\title{
Engkabang fat esters for cosmeceutical formulation.
}

\begin{abstract}
Engkabang fat esters were synthesized from engkabang fat using an enzyme as catalyst. The main composition of the fat esters were oleyl palmitate, oleyl stearate and oleyl oleate. The percentage yield was $93.67 \%$. Ternary phase diagrams systems containing fat esters/surfactant/water were constructed. Several regions appeared in the ternary phase diagrams such as isotropic, homogenous, liquid crystal, two-phase and three-phase regions. Increasing the hydrophilic-lipophilic balance value of the used surfactants gave a larger homogenous and isotropic region in ternary phase diagrams of engkabang fat esters/nonionic surfactant/deionized water. Isotropic and homogenous regions in the ternary phase diagram of engkabang fat esters: PEG-40 hydrogenated castor oil (2:1)/polyoxyethylene(20) sorbitan trioleate/deionized water, was the largest when compared to the other ternary phase diagrams. The isotropic and homogenous region can be used as a medium in formulation of cosmetics and pharmaceutical products such as creams, lotions, balms and lipsticks.
\end{abstract}

Keyword: Fat ester; Non-ionic; Surfactant; Co-surfactant; Phase diagram. 